\title{
Selective Growth of Homo-Epitaxial Ag-Nano-Layer on Tribo-Assisted-Reorientation
}

\author{
Minoru Goto $^{1)^{*}}$, Hiroaki Kobayashi ${ }^{2)}$ and Koichi Akimoto ${ }^{2)}$ \\ ${ }^{1)}$ Department of Mechanical Engineering, Ube National College of Technology \\ 14-1 Tokiwadai-2chome, Ube, Yamaguchi 755-8555, Japan \\ ${ }^{2)}$ Department of Applied Physics, Nagoya University \\ Furo-cho, Chikusa-ku, Nagoya 460-1111, Japan \\ *Corresponding author: mi-goto@ube-k.ac.jp
}

( Manuscript received 21 January 2008; accepted 15 April 2008; published 30 April 2008 )

( Presented at JAST Tribology Conference Saga, September 2007 )

\begin{abstract}
The growth process and orientational degree of incremental-Ag films with a thickness of 5, 50 and $500 \mathrm{~nm}$, respectively, on reoriented Ag (111) surface were studied by in-situ reflection high-energy electron diffraction (RHEED). The orientation of $\mathrm{Ag}$ grains for each incremental-Ag-film was also analyzed by synchrotron-orbital-radiated X-ray diffraction (SOR-XRD) to clarify the correlation between the film orientation and the incremental thickness by additional Ag deposition. RHEED observations showed that the incremental-Ag-film grows epitaxially on the reoriented-Ag (111) surface up to $500 \mathrm{~nm}$ in thickness. The Ag film, which grew on initial polycrystalline-Ag film, still showed a polycrystalline structure up to $50 \mathrm{~nm}$ in thickness, and the Ag (111) orientation is dominant at a thickness of $500 \mathrm{~nm}$. The results of SOR-XRD analysis agreed well with those of RHEED. SOR-XRD analysis showed that the growth rate of Ag $\{111\}$ grains in the polycrystalline film was also superior to that of other Ag $\{\mathrm{hkl}\}$ grains as the thickness increases. The degree of incremental film orientation increased as the incremental thickness increased, and the advantage in the high orientational degree of the incremental film on the reoriented Ag (111) surface was enhanced when the incremental thickness was within the nanometric range. A new method for selective growth of the epitaxial Ag layer using tribo-assisted reorientation was presented.
\end{abstract}

Keywords: Ag film, Si (111), epitaxy, selective growth, RHEED, SOR, XRD, reorientation

\section{Introduction}

The authors have reported that the crystal orientation of a selective area in polycrystalline $\mathrm{Ag}$ film with a thickness of $5 \mathrm{~nm}$ can be changed by a mechanical rubbing process using a mirror-polished diamond sphere in an ultrahigh vacuum (UHV) condition, a process called tribo-assisted reorientation ${ }^{1,2)}$. As a result, the mono-crystal-Ag (111) film region is generated only in a rubbed area within the polycrystalline film on the same substrate. This phenomenological finding suggested the possibility of a new processing technique, by which the crystal orientation of metallic film can be controlled at only a given region by a tribological method. This means that a multi-composite structured Ag film with the two different regions can be readily prepared by the tribo-assisted reorientation phenomenon; one is an $\mathrm{Ag}$ film region of mono-crystalline structure and the other of polycrystalline structure. Moreover, a geometric pattern with a flexible shape is possible.
The tribo-assisted reorientation phenomenon is limited to a thickness of around $5 \mathrm{~nm}$; the phenomenon does not occur with a thick film of 50-nm. The growth process of metallic films is strongly affected by the surface states of the substrate such as chemical composition and/or the atomic arrangement of the topmost layer, as well as the deposition conditions ${ }^{1-8)}$. For example, $\mathrm{Ag}$ (111) film grows epitaxially on an $\mathrm{Si}$ (111) $7 \times 7$ clean surface, whereas a small amount of Ag $\{100\}$ grains is grown on an $\mathrm{Si}(111) \sqrt{3} \times \sqrt{3}-\mathrm{Ag}$ surface together with a large amount of Ag $\{111\}$ grains, i.e., polycrystalline Ag film, in an UHV environment ${ }^{1,2,9,10)}$. If the growth process of $\mathrm{Ag}$ film shows an epitaxial mode on the reoriented-Ag (111) surface, a thicker than 5-nm multi-composite Ag film with the coexisting regions, i.e. initial polycrystalline film region and homo-epitaxial film region can be produced on the same substrate. The mono-crystal pattern region will be, for example, used for beam splitter for X-rays beam with the given geometry of the cross-section, as well as the substrate for a hetero-epitaxial film. Thus, it is 
important to clarify the growth mode of the incremental Ag layer by additional deposition.

The main objective of this paper is to elucidate the growth process and orientational degree of Ag films on the reoriented-Ag (111) surfaces due to tribo-assisted reorientation, as a function of the incremental thickness. This growth process should be elucidated to establish the innovative process of the multi-composite-Ag-film generation. Incremental-Ag films with the thickness of 5, 50 , and $500 \mathrm{~nm}$, respectively, were investigated on the re-oriented $\mathrm{Ag}$ (111) surface of polycrystalline film with a mean thickness of $5 \mathrm{~nm}$.

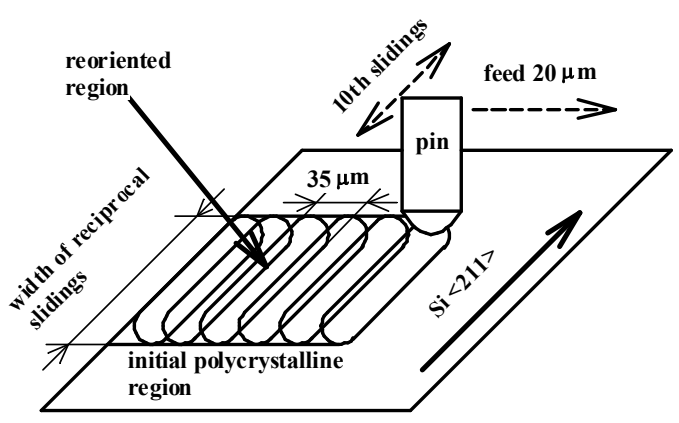

(a) Procedure of reoriented surface preparation
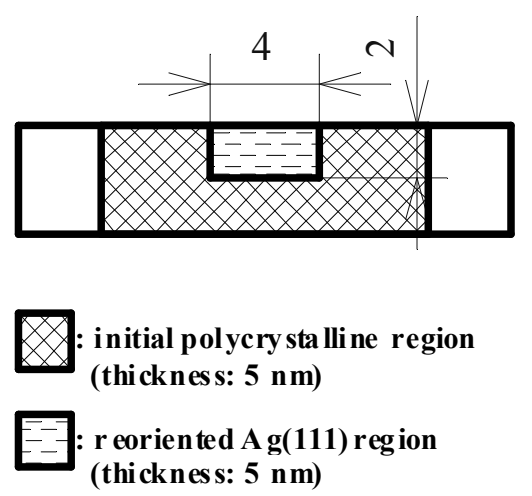

(b) Top view of the dimension of reoriented $\mathrm{Ag}$ (111) region in initial polycrystalline film

Fig.1 Schematic diagrams of reoriented Ag (111) surface preparation within the selective area of $2 \times 4 \mathrm{~mm}^{2}$

\section{Experimental}

The silicon (111) wafer was used as a substrate with the dimensions of $18 \times 4 \times 0.3 \mathrm{~mm}^{3}$. The oxide and/or contaminant layers on the $\mathrm{Si}$ (111) surface of the substrate were removed by iterated flashings under a UHV environment at around $1473 \mathrm{~K}$ via a direct current through the substrate, just before Ag deposition. The Si (111) surface was defined as clean when a clear $7 \times 7$ reflection high-energy electron diffraction (RHEED) pattern was obtained after the flash cleanings ${ }^{4}$. A source of $\mathrm{Ag}$ with a purity of $99.999 \%$ was used for $\mathrm{Ag}$ deposition. The Ag films were prepared on the substrate using a water-cooled Knudsen cell in a UHV condition at a pressure less than $4 \times 10^{-8} \mathrm{~Pa}$. The thickness of the film was controlled by deposition time and deposition rate, and the temperature shift of the $\mathrm{Ag}$ source during deposition was maintained at less than $0.5 \mathrm{~K}$. A spherical surface of single-crystal diamond with radius of curvature of $3 \mathrm{~mm}$ was used as a slider to prepare the reoriented-Ag (111) surface of polycrystalline-Ag film with a thickness of $5 \mathrm{~nm}$ due to the tribo-assisted reorientation. The roughness of the sliding surface was less than $2.6 \mathrm{~nm}$ r.m.s. The sliding surface of the diamond was cleaned with diluted nitric acid to eliminate heavy metallic contamination, then ultrasonically cleaned in acetone before installation in this experimental apparatus.

Prior to generating the reoriented-Ag (111) surface of selective areas, polycrystalline-Ag film with a thickness of $5 \mathrm{~nm}$ was prepared on an $\mathrm{Si}$ (111) $\sqrt{3} \times \sqrt{3}-\mathrm{Ag}$ (hereinafter referred to as $\sqrt{3}-\mathrm{Ag}$ ) surface at a temperature less than $303 \mathrm{~K}$. The $\sqrt{3}-\mathrm{Ag}$ surface was created by heating an atomic layer of $\mathrm{Ag}$ on the $\mathrm{Si}$ (111) $7 \times 7$ surface of the substrate to around $773 \mathrm{~K}$, which was determined by RHEED ${ }^{11)}$. The selective area of the polycrystalline-Ag film was rubbed by the diamond sphere in the UHV condition. The process of reoriented $\mathrm{Ag}$ (111) surface generation within the selective area is shown in Fig. 1. The normal load and sliding speed were $250 \mathrm{mN}$ and $1.0 \mathrm{~mm} / \mathrm{s}$, respectively. The ten reciprocal slidings by the diamond slider were done parallel to the $<211>$ axis of the Si (111) substrate. The worn track width on the film is around $35 \mu \mathrm{m}$ under these sliding conditions, and the length is easily controlled by extending the swinging width of the reciprocal sliding. By overlapping the edge of many worn tracks, a large rubbed region is obtained (see Fig. 1 (a)). The crystal orientation of the polycrystalline film was changed to mono-crystalline film due to tribo-assisted reorientation in the rubbed area. Then, the Si (111) substrate was covered with two kinds of $\mathrm{Ag}$ films having the same 5-nm thickness, i.e. initial polycrystalline-Ag film coexistent with newly-generated reoriented-Ag (111) film, which was ascertained by RHEED ${ }^{1,2)}$. The characteristic diffraction pattern originated from $\mathrm{Ag}$ (111) plane was observed on the rubbed area of the film, whereas no significant spots appeared from initial polycrystalline film ${ }^{1}$. The reoriented area of $4 \times 2 \mathrm{~mm}^{2}$ was generated in the initial polycrystalline film, and was used as the new substrate for the deposition of incremental-Ag film (see Fig. 1 (b)). The incremental thicknesses of 5, 50 and $500 \mathrm{~nm}$, which were deposited using water-cooled Knudsen cell in the same UHV condition, were investigated in this study. The details of the experimental apparatus and the sample preparations are reported elsewhere ${ }^{1,2,11)}$.

The crystal orientations on the surface of the 
incremental films deposited on the reoriented-Ag (111) regions were observed by in-situ RHEED as a function of film thickness, and obtained data were compared with those of incremental-Ag film grown on the initial polycrystalline-Ag region. The incident angle of the beam was less than $4^{\circ}$, and the incident direction of the beam against the $\mathrm{Si}(111)$ substrate was $<110>$. The accelerating voltage of the incident beam was $15 \mathrm{kV}$. The orientations of the incremental-Ag films were analyzed by synchrotron-orbital-radiated X-ray diffraction (SOR-XRD) in an ambient atmosphere. SOR-XRD observations were performed with the approval of the Photon Factory at the High Energy Accelerator Research Organization (KEK) in Japan. A BL-9C (beam size: $1 \times 1$ $\mathrm{mm}^{2}$ ) beam line with a wavelength of $0.1218 \mathrm{~nm}$ was selected to analyze the orientational degree of the incremental-Ag films. The experimental configuration of SOR-XRD was reported in the previous work ${ }^{1,2,9,10}$. The relative intensity ratio between $\mathrm{Ag}$ (111) diffraction intensity $\left(\mathrm{I}_{\mathrm{Ag}(111)}\right)$ and $\mathrm{Ag}$ (200) diffraction intensity $\left(\mathrm{I}_{\mathrm{Ag}(200)}\right)$ on a $\theta-2 \theta$ method was characterized by the orientational degree of the incremental films.

\section{Results and discussion}

3.1. Selective growth mode of homo-epitaxial Ag film on reoriented $\mathrm{Ag}$ (111) surface

Figure 2 shows the RHEED pattern obtained from the incremental film with $5 \mathrm{~nm}$ thickness deposited on (a) initial polycrystalline and (b) reoriented Ag (111) surfaces, respectively. The characteristic RHEED pattern of the incremental film on the reoriented surface originated from the $\mathrm{Ag}$ (111) plane, whereas such a pattern was not observed on another part of the incremental film deposited on the initial polycrystalline surface. This means that the incremental Ag film grew epitaxially on the selective region of reoriented $\mathrm{Ag}$ (111) surface due to tribo-assisted reorientation, and that the incremental Ag film grew as a polycrystalline structure on the initial polycrystalline surface. Selective growth of homo-epitaxial film on the reoriented surface occurs when the incremental thickness increases to $50 \mathrm{~nm}$ by additional deposition.

On the other hand, the characteristic RHEED pattern of $\mathrm{Ag}$ (111) plane was also observed on the incremental film of $500 \mathrm{~nm}$ thickness deposited on the initial polycrystalline surface, as well as the pattern of the film grown on the reoriented surface (see Fig. 3). Thus, it was concluded that the selective growth of homo-epitaxial film on reoriented $\mathrm{Ag}$ (111) region characteristically occurred at the incremental thickness less than $50 \mathrm{~nm}$, judging from the result of RHEED.

However, the diffraction spots from the 500-nm incremental film on initial polycrystalline surface were slightly diffuse compared with the spots from the film on the reoriented surface. This means that the orientational degree of both films, i.e. 500-nm incremental film on initial polycrystalline surface and that on the reoriented surface, was different, and the quantitative analysis by SOR-XRD was presented in section 3.2 .

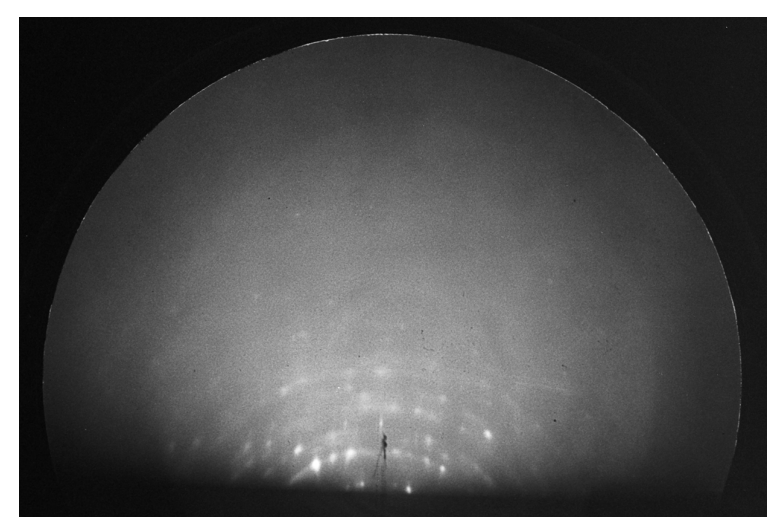

(a) incremental film on initial polycrystalline surface

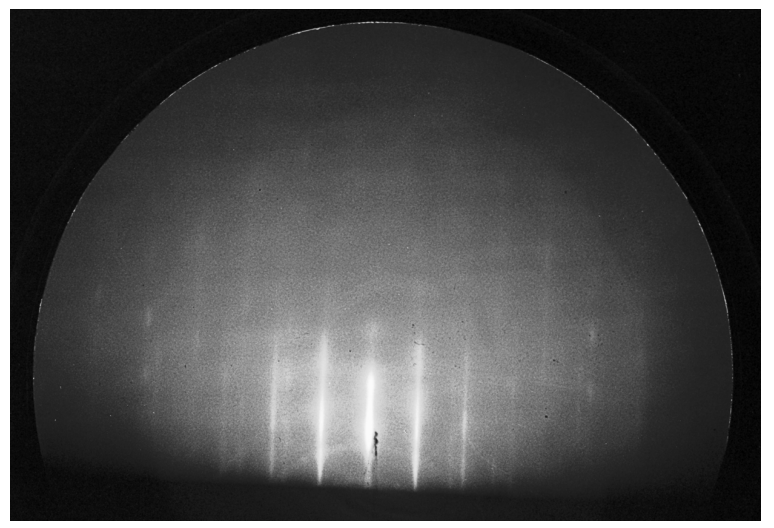

(b) incremental film on reoriented $\mathrm{Ag}$ (111) surface

Fig.2 RHEED pattern of incremental film with 5 $\mathrm{nm}$ thickness deposited on (a) initial polycrystalline and (b) reoriented surfaces

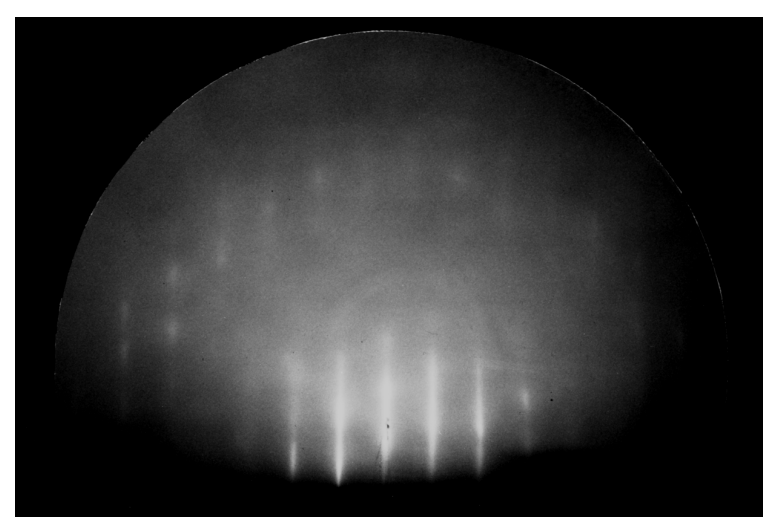

Fig.3 RHEED pattern of incremental film with $500 \mathrm{~nm}$ thickness deposited on initial polycrystalline surfaces 
3.2. Orientation change of $\mathrm{Ag}$ films on two kinds of surface

Figure 4 shows the transition of the intensity ratio between $\mathrm{Ag}$ (111) and $\mathrm{Ag}$ (200) diffraction of X-rays as a function of the incremental thickness obtained by SOR-XRD. The increase in $\mathrm{I}_{\mathrm{Ag}(111)} / \mathrm{I}_{\mathrm{Ag}(200)}$ defined here is the increase in the orientational degree, which means the ratio of $\mathrm{Ag}\{111\}$ and the total number of $\mathrm{Ag}\{\mathrm{hkl}\}$ grains in the film. The highly-orientated Ag (111) films were grown on the reoriented Ag (111) surface for the entire range of incremental thickness, compared with the film grown on the initial polycrystalline surface. The advantage in the high orientational degree of the incremental film on the reoriented Ag (111) surface was enhanced when the incremental thickness was in the nanometric range. With an incremental thickness of 5 $\mathrm{nm}$, the orientational degree of the film on the reoriented surface was more than ten times larger than that on the initial polycrystalline surface.

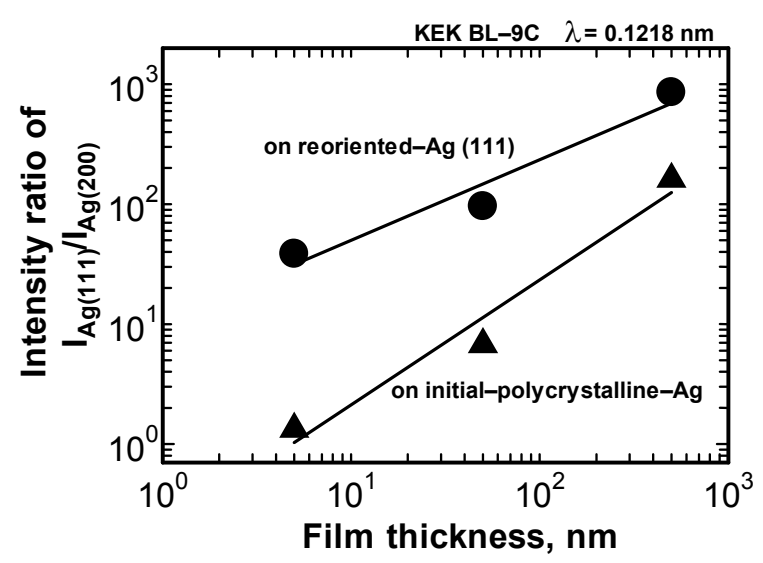

Fig.4 Relationship between intensity ratio of $\mathrm{I}_{\mathrm{Ag}(111)} / \mathrm{I}_{\mathrm{Ag}(200)}$ and incremental thickness

\section{Conclusions}

The growth process and orientational degree of incremental-Ag-films with a thickness of 5, 50 and 500 $\mathrm{nm}$, respectively, were studied on two kinds of surfaces, i.e. reoriented-Ag (111) surface and initial polycrystalline surface. The conclusions may be summarized as follows.

(1) The growth mode of incremental Ag film shows epitaxial growth on the selective area of the reoriented Ag (111) surface of polycrystalline film due to tribo-assisted reorientation.

(2) The orientational degree of incremental film increased as the incremental thickness increased, and the advantage of the high orientational degree of the incremental film on the reoriented $\mathrm{Ag}$ (111) surface was enhanced when the incremental thickness was in the nanometric range.

(3) With an incremental thickness of $5 \mathrm{~nm}$, the orientational degree of the film on the reoriented surface was more than ten times larger than that on the initial polycrystalline surface.

\section{Acknowledgment}

This work was financed in part by a research grant from The Mazda Foundation, and by a Grant-in-Aid for Scientific Research (No. 19560154) from the Ministry of Education, Culture, Sports, Science and Technology, Japan.

\section{References}

[1] Goto, M., Akimoto, K., Honda, F. and Nakahara, T., "Tribo-Assisted Reorientation of Nanometer-Thick Ag Film in Ultrahigh Vacuum Environment," Proc. Instn. Mech. Engrs., Part J: J. Engineering Tribology, 220, 3, 2006, 135-142.

[2] Akimoto, K., Fukagawa, K., Goto, M. and Honda, F., "Crystal Orientation Changes of Ag Thin Films on the $\mathrm{Si}(111)$ Substrate Due to Tribo-Assisted Recrystallization," Thin Solid Films, 515, 2006, 444-447.

[3] Nagakura, S., Wakashima, K. and Fukamachi, M., "Structure and Tensile Properties of Thick Single-Crystal Silver Films Formed by Vacuum Deposition," J. Vac. Sci. Technol., 6, 4, 1969, 611-614.

[4] Gotoh, Y. and Ino, S.,"Surface Structure of Ag on Si (111) Surface Investigated by RHEED," Jpn. J. Appl. Phys., 17, 12, 1978, 2097-2109.

[5] Le Lay, G., "Physics and Electronics of the Noble-Metal/Elemental-Semiconductor Interface Formation: A Status Report," Surf. Sci., 132, 1983, 169-204.

[6] Gavioli, L., Kimberlin, K. R., Tringides, M. C., Wnedelken, J. F. and Zhang, Z., "Novel Growth of Ag Islands on Si (111): Plateaus with a Singular Height," Phys. Rev. Lett., 82, 1, 1999, 129-132.

[7] Galiana, N., Martin, P. P., Munuera, C., Varela, M., Soria, F., Ocal, C., Ruiz, A. and Alonso, M., "MBE Fabrication of Self-Assembled $\mathrm{Si}$ and Metal Nanostructures on Si Sufaces," Surf. Sci., 600, 2006, 3956-3963.

[8] Goswami, D. K., Bhattacharjee, K., Stapati, B., Roy, S., Satyam, P. V. and Dev, B. N., "Preferential Heights in the Growth of Ag Islands on Si(111)(7×7) Surfaces," Surf. Sci., 601, 2007, 603-608.

[9] Emoto, T., Akimoto, K., Ishikawa, Y. and Ichimiya, A., "Quantitative Evaluation of Near Reconstructed Si Surface," Surf. Sci., 493, 2001, 221-226.

[10] Emoto, T., Akimoto, K., Ichimiya, A. and Hirose, K., "Strain Due to Nickel Diffusion into Hydrogen-Terminated Si(111) Surface," Appl. Surf. Sci., 190, 2002, 113-120.

[11] Goto, M., Nakata, R. and Honda, F., "Tribological Behavior of an Ag Sub-Nanometer-Thick Film on an Si (111) 7×7 Surface at Elevated Temperatures," Wear, 256, 2004, 726-734. 